\title{
Hydrodynamics of an airlift reactor for aeration in molten sulfur
}

\author{
Junjie Wang ${ }^{1}$, Xiao Xu², Qiang Yang ${ }^{2}$, Wei Wang ${ }^{2}$, Yudong $\mathrm{Li}^{2}$, Shihan $\mathrm{Wu}^{2}$, and Haiqiang \\ Yang $^{2}$ \\ ${ }^{1}$ East China University of Science and Technology \\ ${ }^{2}$ Affiliation not available
}

April 6, 2021

\begin{abstract}
An industrial-scale internal loop airlift reactor is used to remove volatile gas from high-viscosity molten sulfur. The effects of the superficial gas velocity and reactor height on the hydrodynamic characteristics were studied. The gas holdup, average bubble diameter, and liquid circulation velocity in the reactor under different conditions were analyzed using computational fluid dynamics simulation. The superficial gas velocity was varied from $0.0056 \mathrm{~m} / \mathrm{s}$ to $0.05 \mathrm{~m} / \mathrm{s}$ at a constant reactor height of $15 \mathrm{~m}$. The total reactor height was varied from $5 \mathrm{~m}$ to $25 \mathrm{~m}$ at a superficial gas velocity of $0.0389 \mathrm{~m} / \mathrm{s}$. Based on the correlation between the gas holdup and liquid circulation velocity proposed by Chisti (1988), an optimized correlation between the gas holdup and liquid circulation velocity was developed by considering the influence of the bubble diameter. The results obtained using the proposed correlation were compared with those obtained using the Chisti correlation and simulation.
\end{abstract}

\section{Hosted file}

Hydrodynamics of an airlift reactor for aeration in molten sulfur-manuscript.pdf available at https://authorea.com/users/358394/articles/516926-hydrodynamics-of-an-airlift-reactorfor-aeration-in-molten-sulfur 


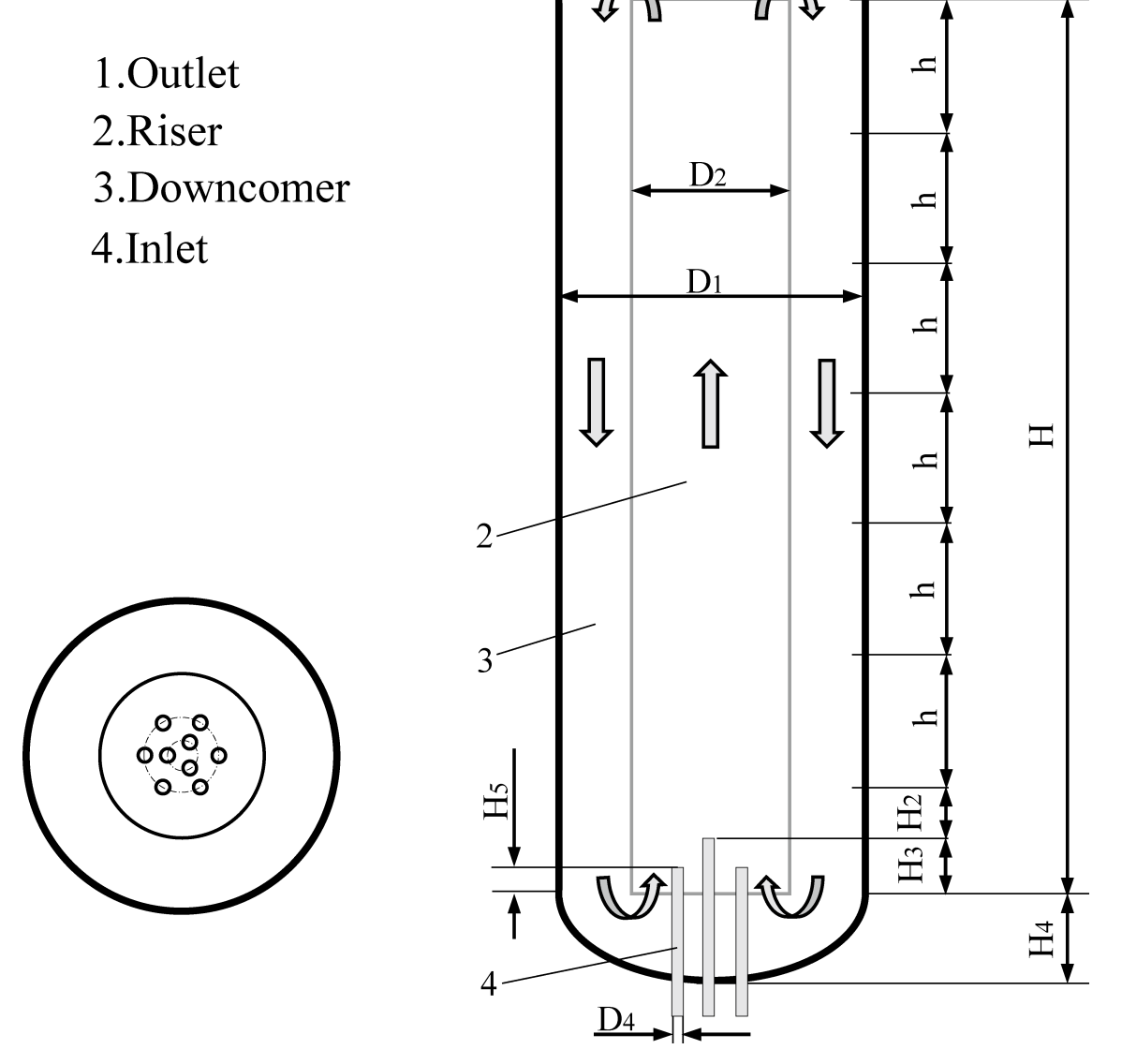


Gas holdup

$\left[\begin{array}{c}1.000 \mathrm{e}-001 \\ 9.000 \mathrm{e}-002 \\ 8.000 \mathrm{e}-002 \\ 7.000 \mathrm{e}-002 \\ 6.000 \mathrm{e}-002 \\ 5.000 \mathrm{e}-002 \\ 4.000 \mathrm{e}-002 \\ 3.000 \mathrm{e}-002 \\ 2.000 \mathrm{e}-002 \\ 1.000 \mathrm{e}-002 \\ 0.000 \mathrm{e}+000\end{array}\right.$
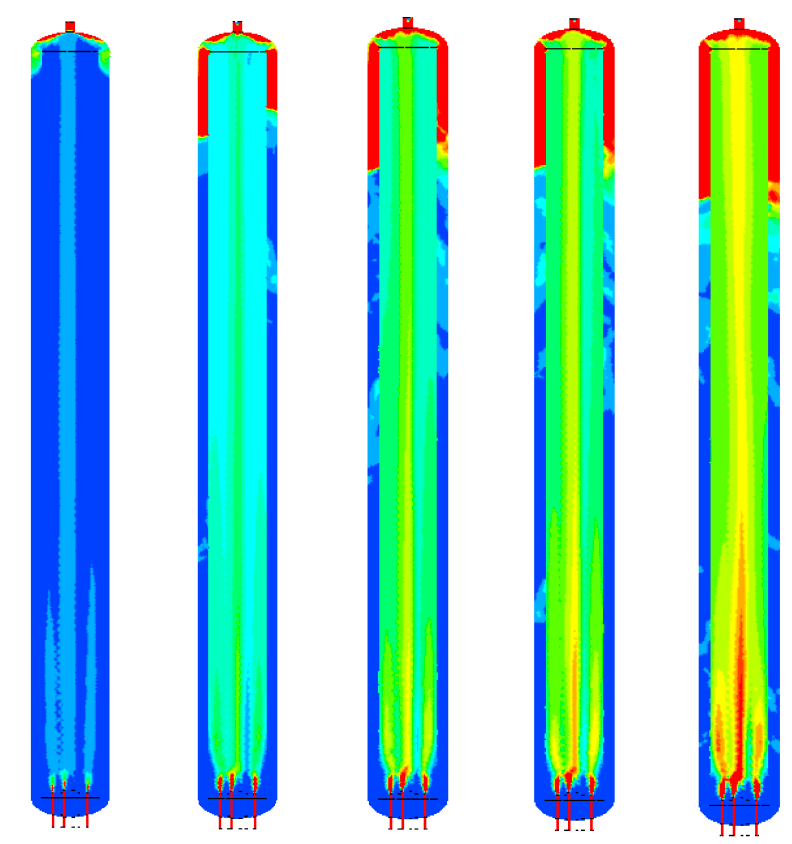

$U_{G r}=0.0056 \mathrm{~m} / \mathrm{s} \quad U_{G r}=0.0222 \mathrm{~m} / \mathrm{s} \quad U_{G r}=0.0333 \mathrm{~m} / \mathrm{s} \quad U_{G r}=0.0389 \mathrm{~m} / \mathrm{s} \quad U_{G r}=0.0500 \mathrm{~m} / \mathrm{s}$

\section{Gas holdup}

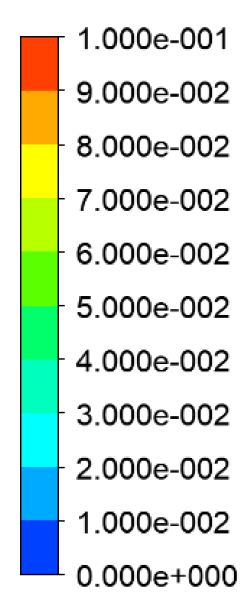

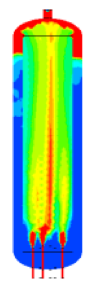

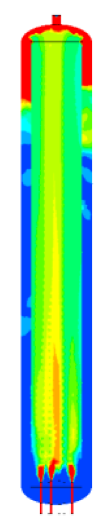

5 m

$10 \mathrm{~m}$
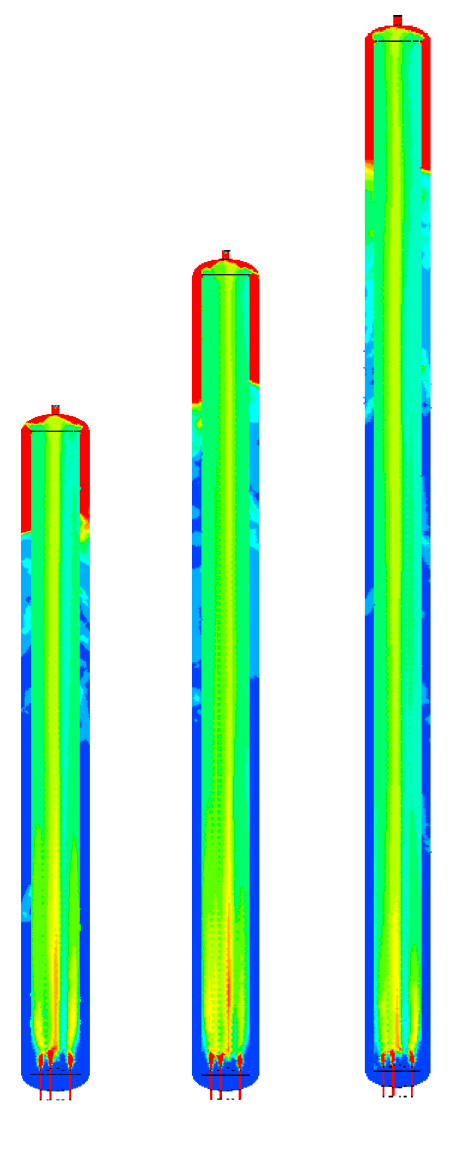

$15 \mathrm{~m}$

$20 \mathrm{~m}$

25 m 

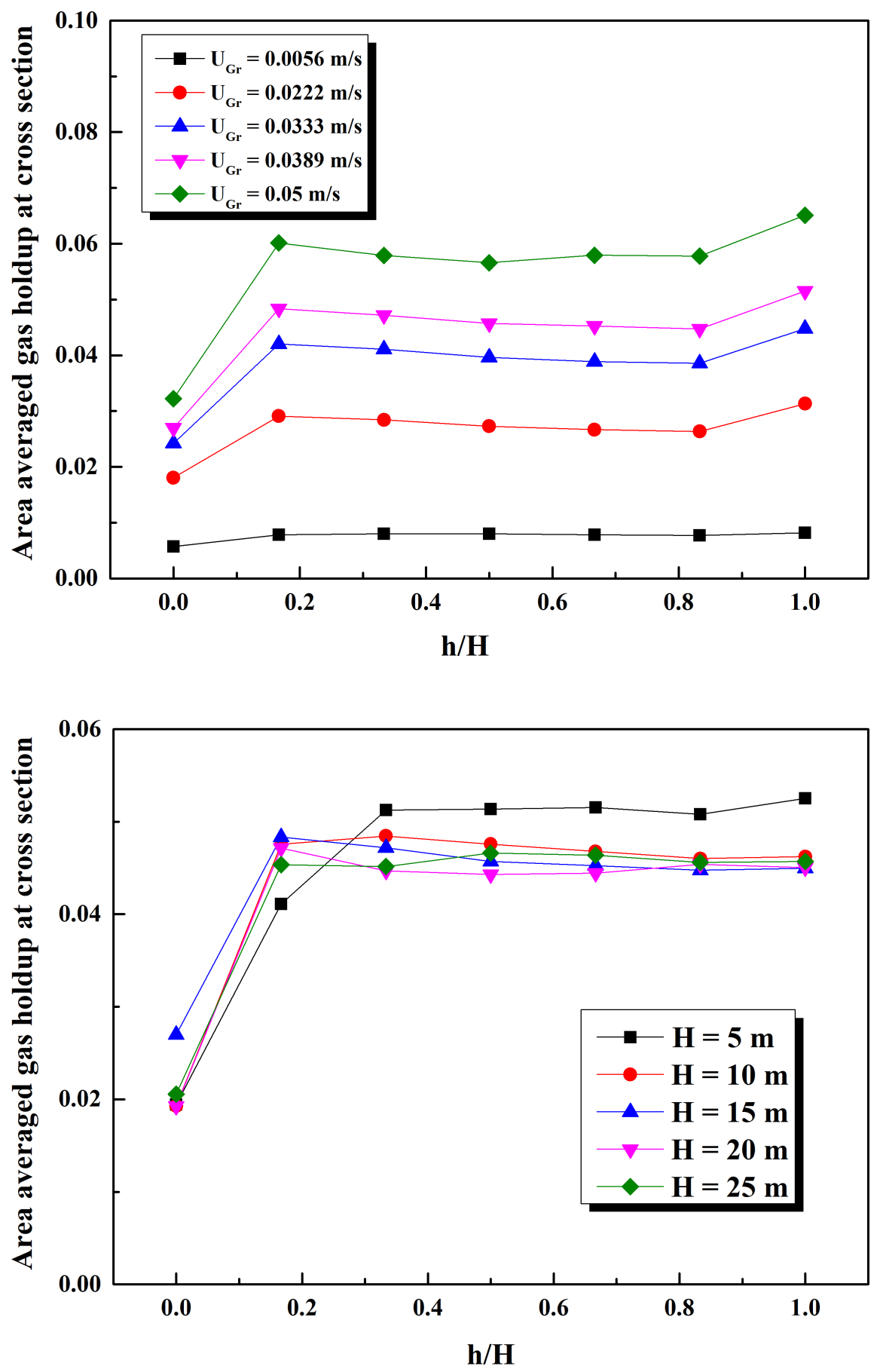

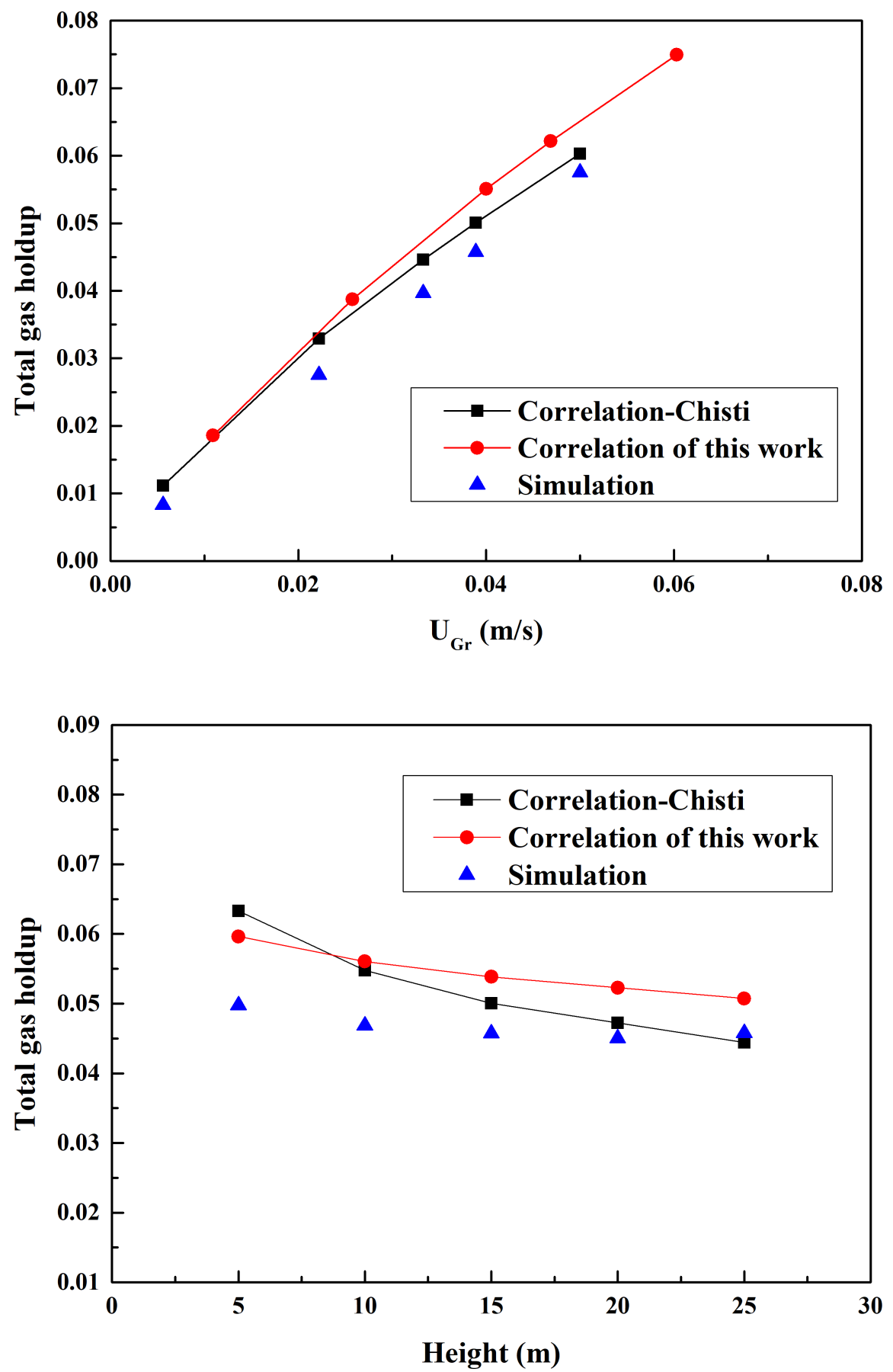


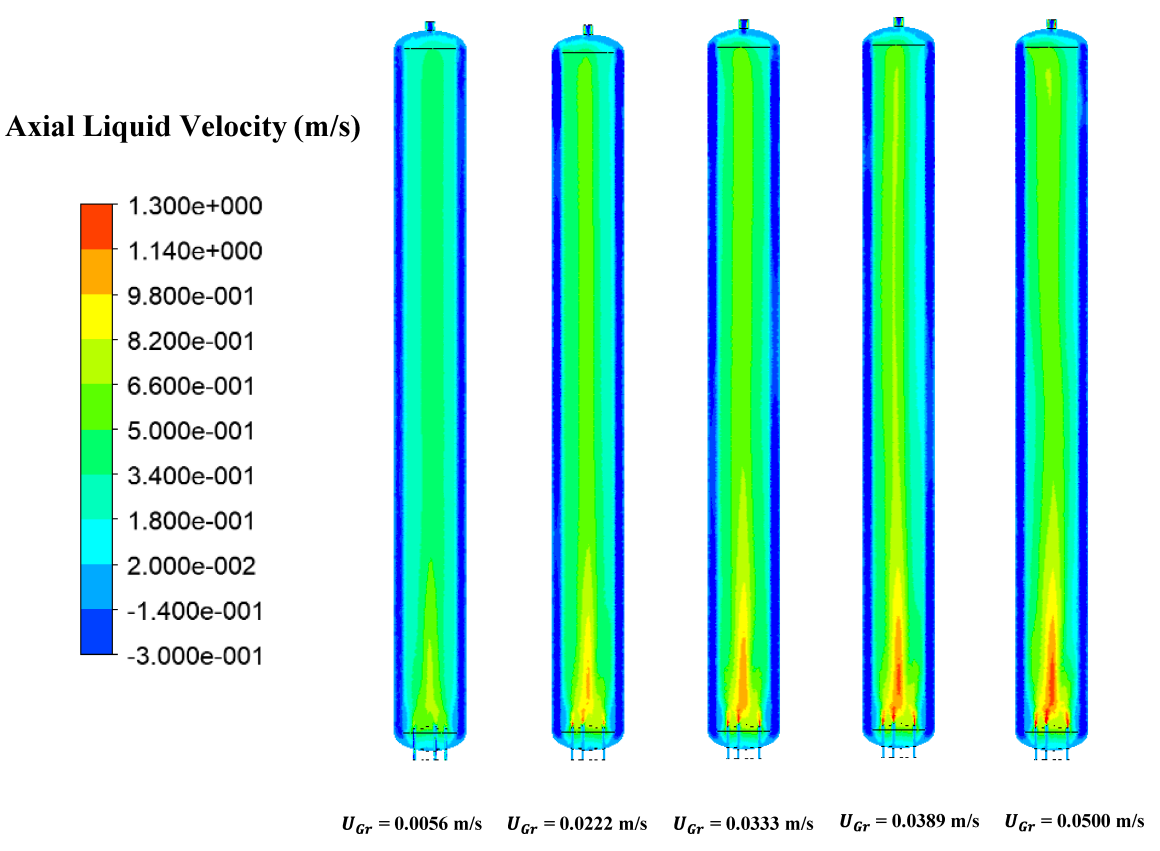

Axial Liquid Velocity (m/s)
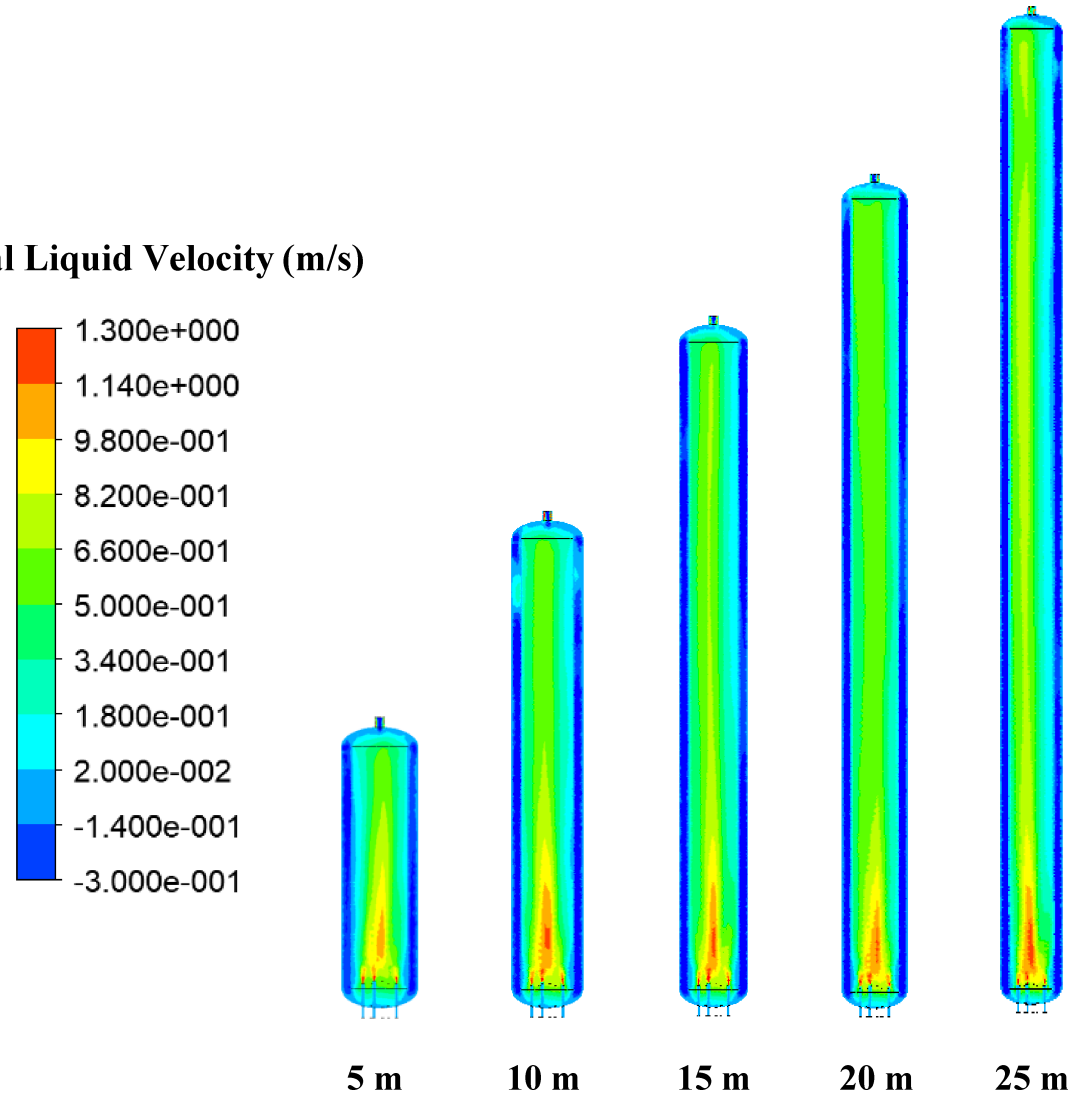

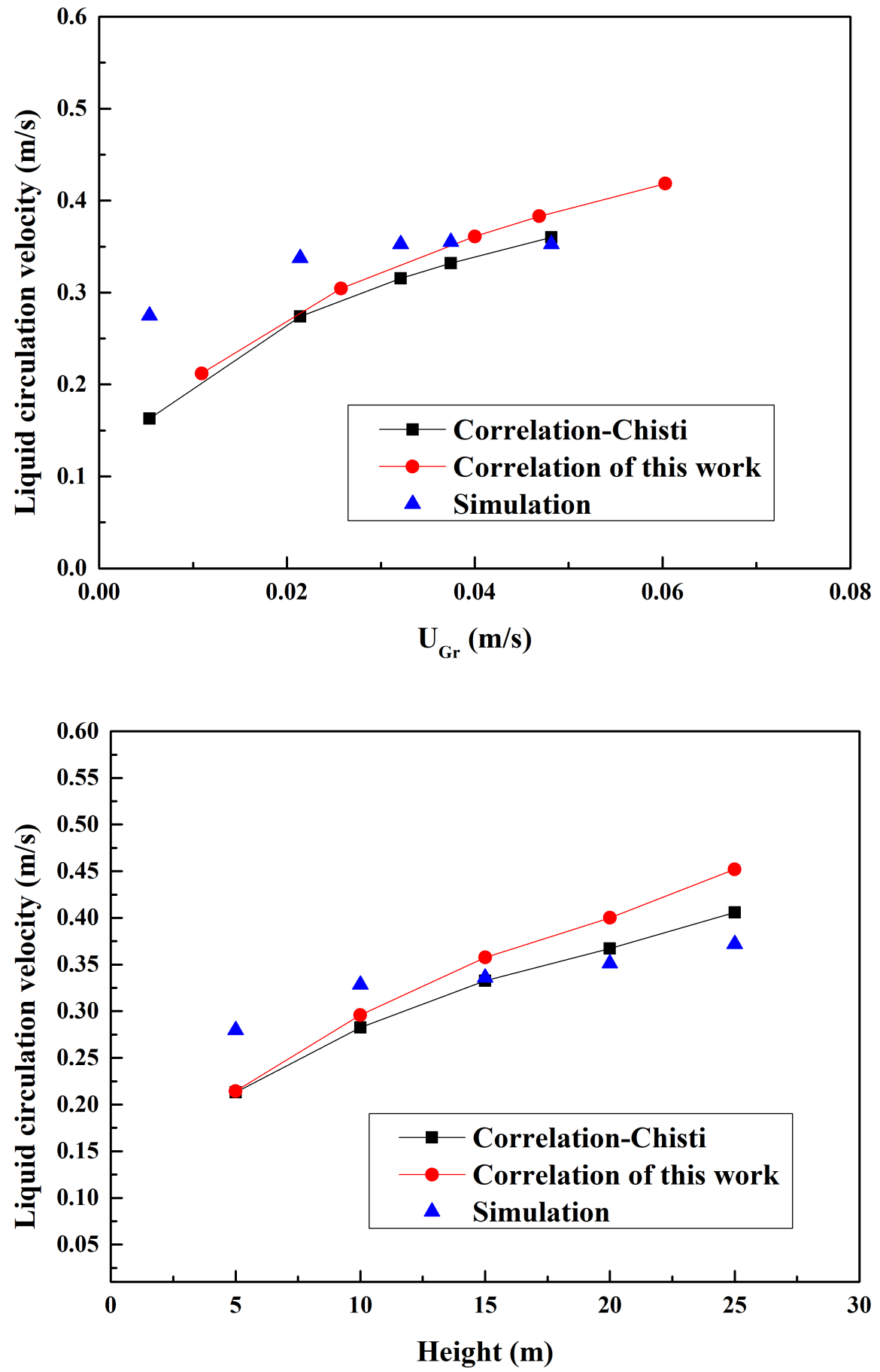


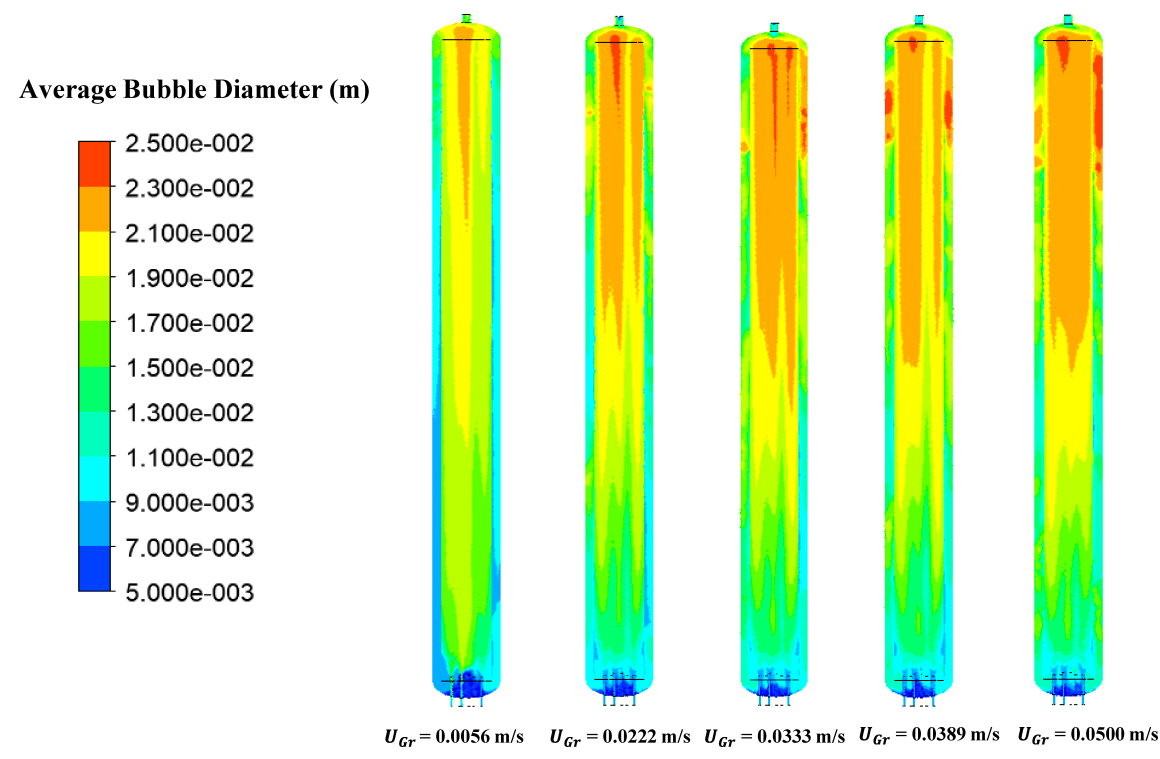

Average Bubble Diameter (m)

$2.500 \mathrm{e}-002$
$2.300 \mathrm{e}-002$
$2.100 \mathrm{e}-002$
$1.900 \mathrm{e}-002$
$1.700 \mathrm{e}-002$
$1.500 \mathrm{e}-002$
$1.300 \mathrm{e}-002$
$1.100 \mathrm{e}-002$
$9.000 \mathrm{e}-003$
$7.000 \mathrm{e}-003$
$5.000 \mathrm{e}-003$
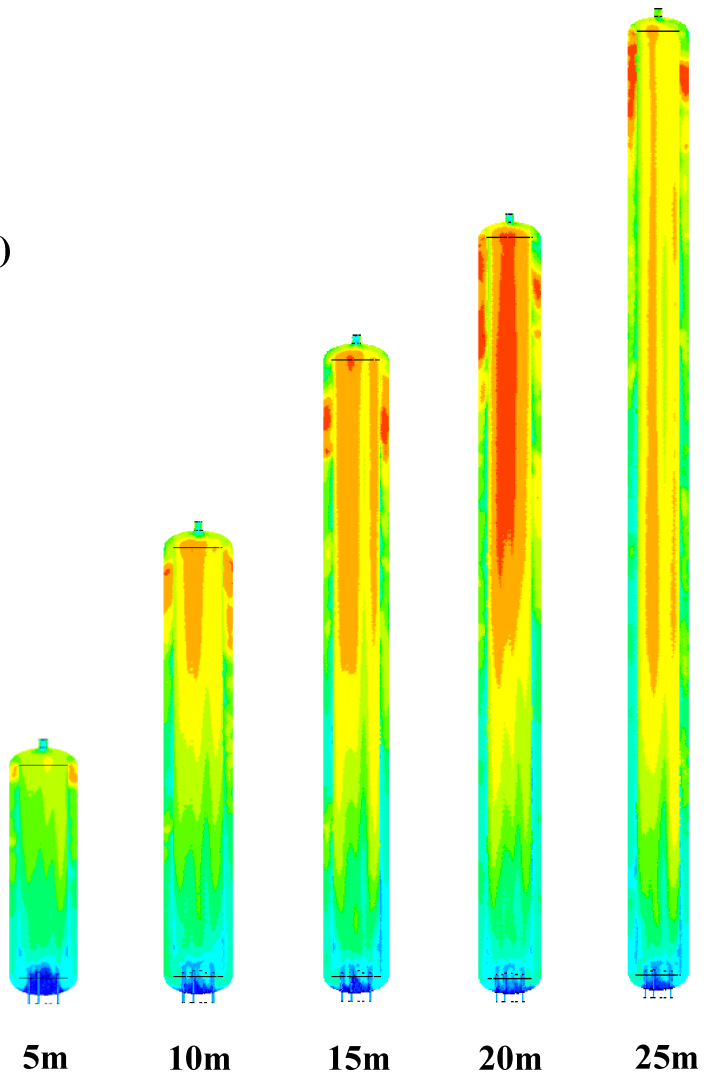

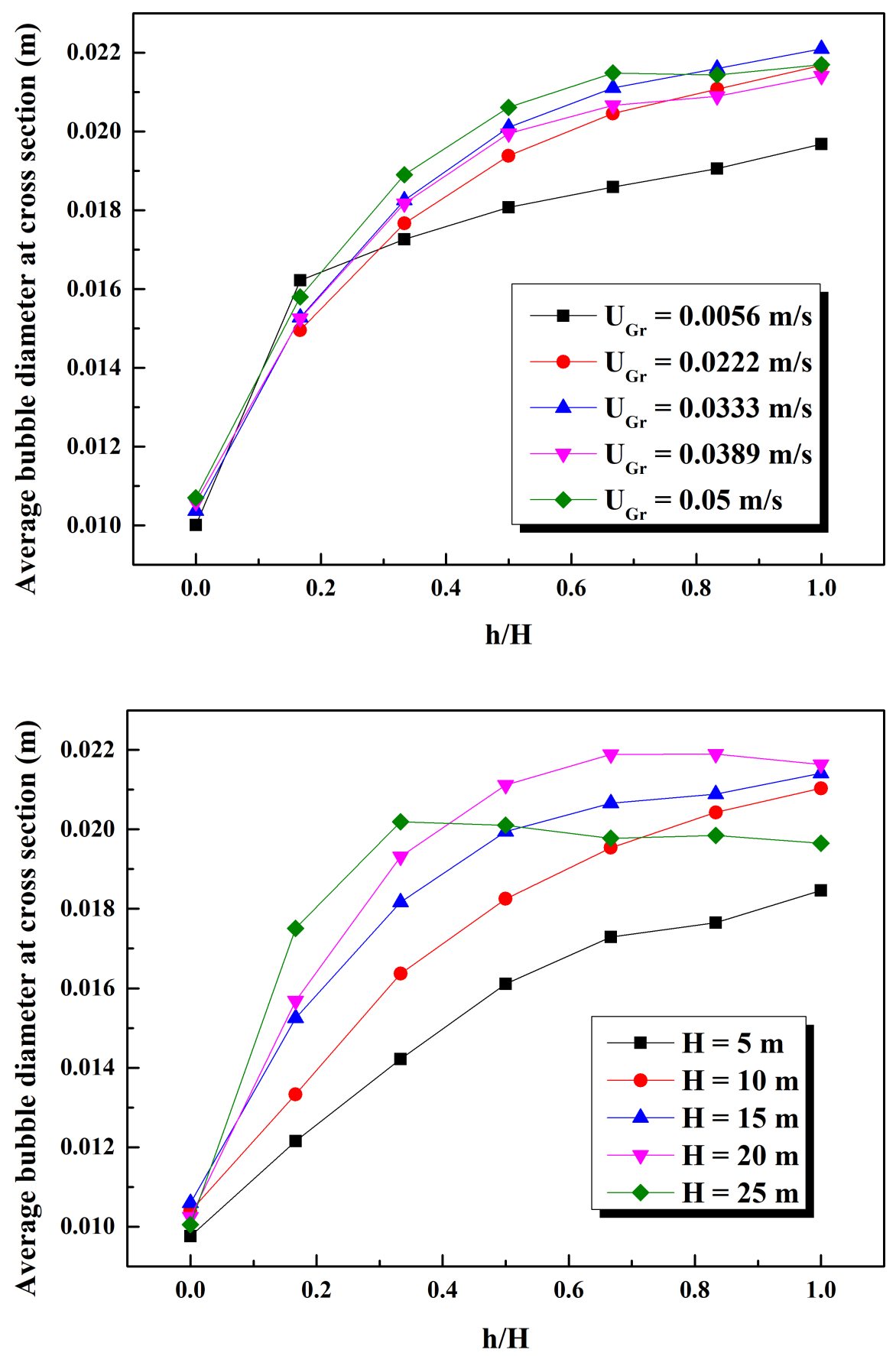


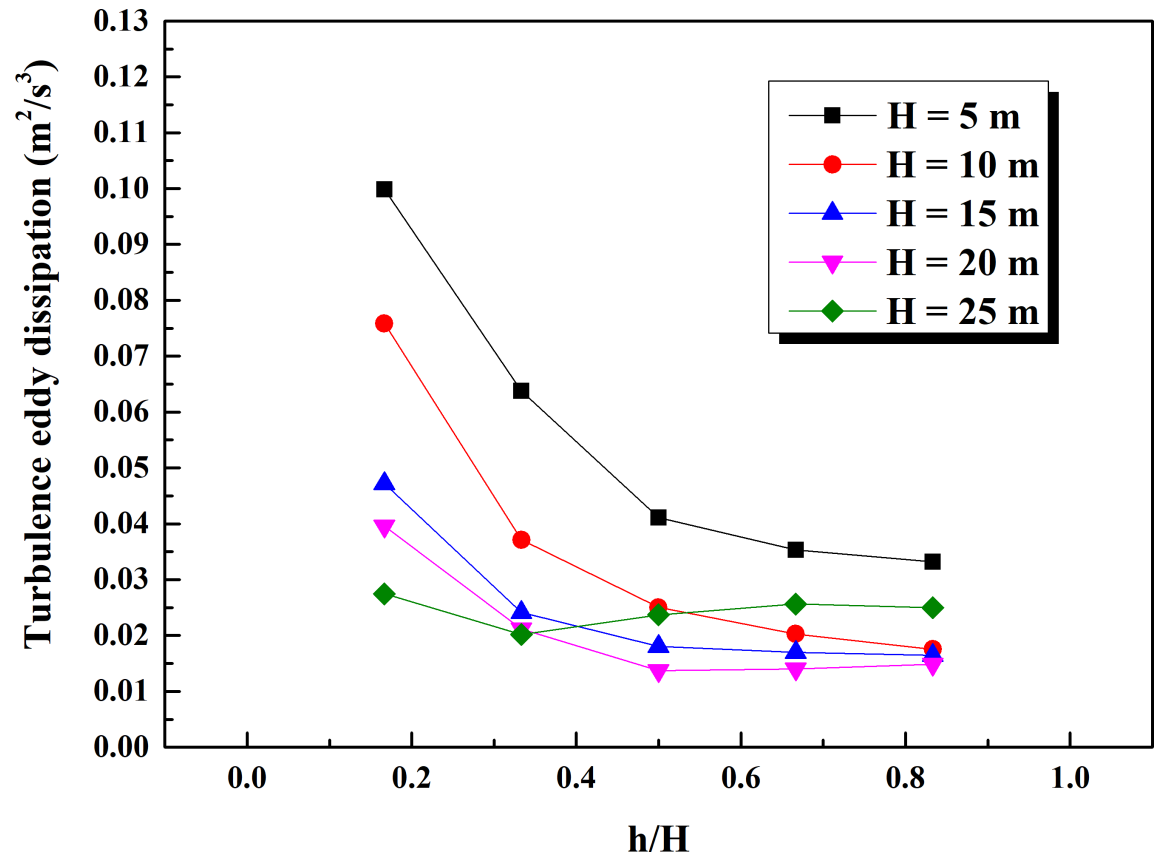

